\title{
Die design for stamping a notebook case with magnesium alloy sheets
}

\author{
Heng-Kuang Tsai, Chien-Chin Liao, Fuh-Kuo Chen* \\ Department of Mechanical Engineering, National Taiwan University, Taipei, Taiwan, ROC
}

\section{A R T I C L E I N F O}

\section{Keywords:}

Notebook case

LZ91 magnesium-lithium alloy

sheet

Multi-operation stamping

Formability

\begin{abstract}
A B S T R A C T
In the present study, the stamping process for manufacturing a notebook top cover case with LZ91 magnesium-lithium alloy sheet at room temperature was examined using both the experimental approach and the finite element analysis. A four-operation stamping process was developed to eliminate both the fracture and wrinkle defects occurred in the stamping process of the top cover case. In order to validate the finite element analysis, an actual four-operation stamping process was conducted with the use of $0.6 \mathrm{~mm}$ thick LZ91 sheet as the blank. A good agreement in the thickness distribution at various locations between the experimental data and the finite element results confirmed the accuracy and efficiency of the finite element analysis. The superior formability of LZ91 sheet at room temperature was also demonstrated in the present study by successful manufacturing of the notebook top cover case. The proposed four-operation process lends itself to an efficient approach to form the hinge in the notebook with less number of operational procedures than that required in the current practice. It also confirms that the notebook cover cases can be produced with LZ91 magnesium alloy sheet by the stamping process. It provides an alternative to the electronics industry in the application of magnesium alloys.
\end{abstract}

(c) 2007 Elsevier B.V. All rights reserved.

\section{Introduction}

Due to its lightweight and good performance in EMI resistance, magnesium alloy has been widely used for structural components in the electronics industry, such as cellular phones and notebook cases. Although the prevailing manufacturing process of magnesium alloy products has been die casting, the stamping of magnesium alloy sheet has drawn interests from industry because of its competitive productivity and performance in the effective production of thin-walled structural components. As for stamping process, AZ31 magnesium alloy (aluminum 3\%, zinc 1\%) sheet has been commonly used for the forming process at the present time, even though it needs to be formed at elevated temperature due to its hexagonal closed-packed (HCP) crystal structure (Chen et al., 2003; Chen and Huang, 2003). Recently, the magnesium-lithium (LZ) alloy has also been successfully developed to improve the formability of magnesium alloy at room temperature. The ductility of magnesium alloy can be improved with the addition of lithium that develops the formation of body centered-cubic (BCC) crystal structure (Takuda et al., 1999a,b; Drozd et al., 2004).

In the present study, the stamping process of a notebook top cover case with the use of LZ sheet was examined. The forming of the two hinges in the top cover of a notebook, as shown in Fig. 1( $a$ and b), is the most difficult operation in the stamping process due to the small distance between the flanges and the small corner radii at the flanges, as displayed in Fig. 1(c). This geometric complexity was caused by a dramatic change in the corner radius when the flange of hinge

\footnotetext{
* Corresponding author.

E-mail address: fkchen@ntu.edu.tw (F.-K. Chen). 


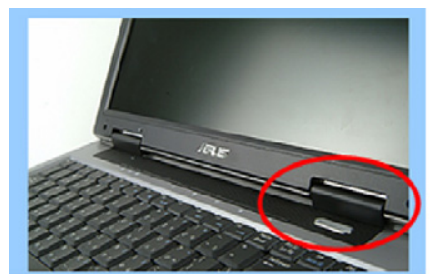

(a)

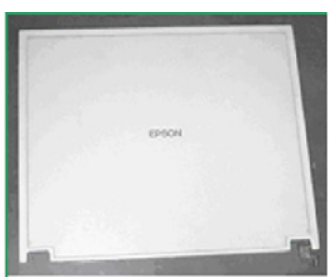

(b)

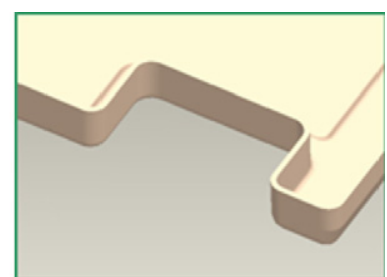

(c)

Fig. 1 - Flange of hinges at notebook top cover case. (a) Hinge, (b) top cover case and (c) flanges of hinge.

gets too close to the edge of the notebook, which would easily cause fracture defect around the flange of hinge and require a multi-operation stamping process to overcome this problem. In the present study, the formability of LZ magnesium alloy sheets was investigated and an optimum multi-operation stamping process was developed to reduce the number of operational procedures using both the experimental approach and the finite element analysis.

\section{Mechanical properties of magnesium alloy sheets}

The tensile tests were performed for magnesium-lithium alloy sheets of LZ61 (lithium 6\%, zinc 1\%), LZ91, and LZ101 at room temperature to compare their mechanical properties to those of AZ31 sheets at elevated temperatures. Fig. 2(a) shows the stress-strain relations of LZ sheets at room temperature and those of AZ31 sheets at both room temperature and $200^{\circ} \mathrm{C}$. It is noted that the stress-strain curve tends to be lower as the
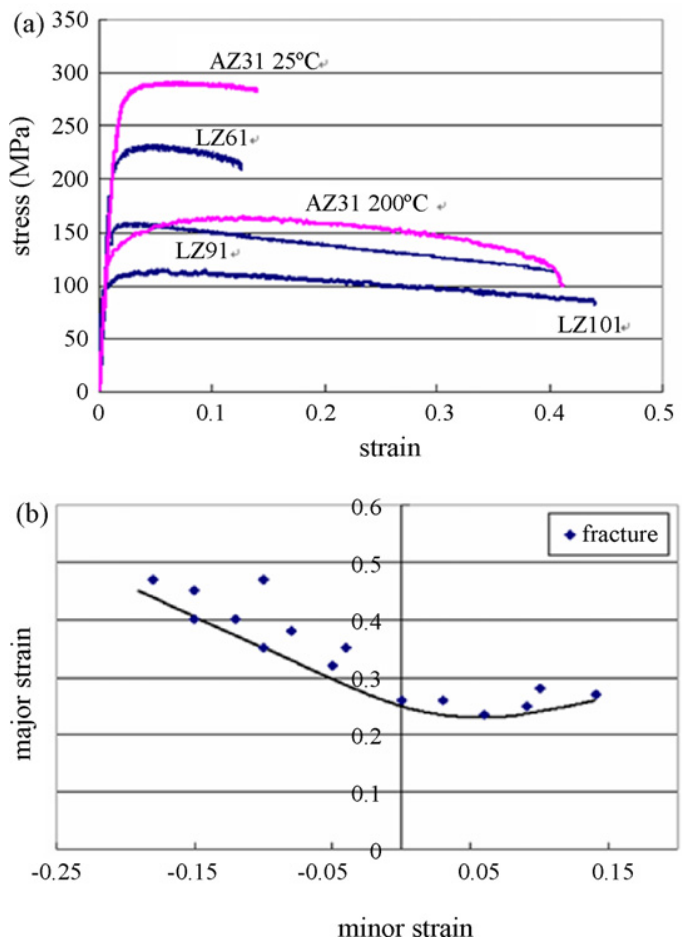

Fig. 2 - Mechanical properties of magnesium alloy. (a) The stress-strain relations of magnesium alloy; (b) forming limit diagram (FLD) of LZ91 sheet. content of lithium increases. It is also observed from Fig. 2(a) that the curves of LZ91 sheet at room temperature and AZ31 sheet at $200^{\circ} \mathrm{C}$ are close to each other. LZ101 sheet at room temperature exhibits even better ductility than LZ91 and AZ31 do at $200^{\circ} \mathrm{C}$. Since the cost of lithium is very expensive, LZ91 sheet, instead of LZ101 sheet, can be considered as a suitable LZ magnesium alloy sheet to render favorable formability at room temperature. For this reason, the present study adopted LZ91 sheet as the blank for the notebook top cover case and attempted to examine the formability of LZ91 at room temperature. In order to determine if the fracture would occur in the finite element analysis, the forming limit diagram for the $0.6 \mathrm{~mm}$ thick LZ91 sheet was also established as shown in Fig. 2(b).

\section{The finite element model}

The tooling geometries were constructed by a CAD software, $\mathrm{PRO} / \mathrm{E}$, and were converted into the finite element mesh, as shown in Fig. 3(a), using the software DELTAMESH. The tooling was treated as rigid bodies, and the four-node shell element was adopted to construct the mesh for blank. The material properties and forming limit diagrams obtained from the experiments were used in the finite element simulations. The other simulation parameters used in the initial run were: punch velocity of $5 \mathrm{~mm} / \mathrm{s}$, blank-holder force of $3 \mathrm{kN}$, and Coulomb friction coefficient of 0.1 . The finite element software PAM_STAMP was employed to perform the analysis, and the simulations were performed on a desktop PC.

A finite element model was first constructed to examine the one-operation forming process of the hinge. Due to symmetry, only one half of the top cover case was simulated, as shown in Fig. 3(a). The simulation result, as shown in Fig. 3(b), indicates that fracture occurs at the corners of flanges, and the minimum thickness is less than $0.35 \mathrm{~mm}$. It implies that the fracture problem is very serious and may not be solved just by enlarging the corner radii at the flanges. The finite element simulations were performed to study the parameters that affect the occurrence of fracture. Several approaches were proposed to avoid the fracture as well.

\section{Multi-operation stamping process design}

In order to avoid the occurrence of fracture, a multi-operation stamping process is required. In the current industrial practice, it usually takes at least ten operational procedures to form the top cover case using the magnesium alloy sheet. In the 


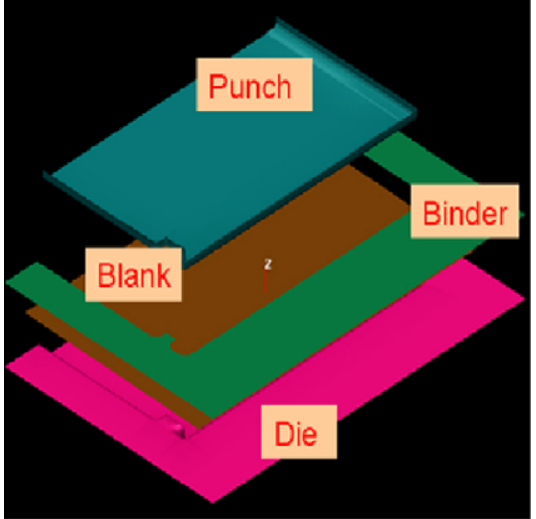

(a)

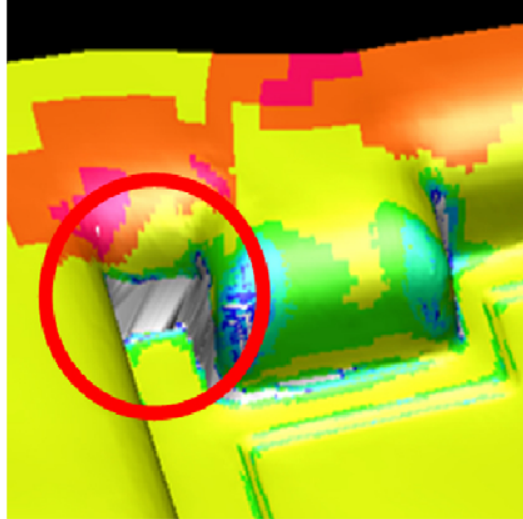

(b)

Fig. 3 - The finite element simulations. (a) Finite element mesh and (b) fracture at the corners.

present study, attempts were made to reduce the number of operational procedures. Several approaches were proposed to avoid the fracture, and the four-operation stamping process had demonstrated itself as a feasible solution to the fracture problem. To limit the length of this paper, only the twooperation and the four-operation stamping processes were depicted in the following.

\subsection{Two-operation stamping process}

The first operation in the two-operation stamping process was sidewall forming as shown in Fig. 4(a), and the second one was

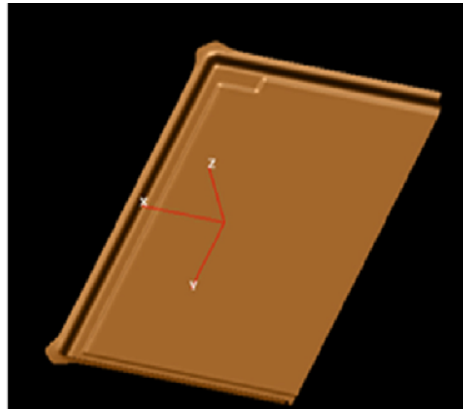

(a)

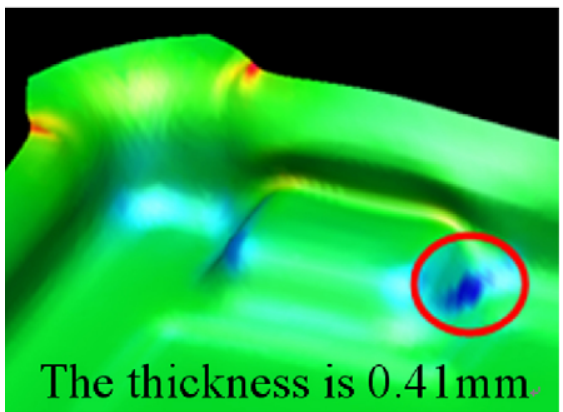

(c) the forming of flange of hinge presented in Fig. 4(b), the height of the flange of hinge being $5 \mathrm{~mm}$. Fig. 4(c) shows the thickness distribution obtained from the finite element simulation. The minimum thickness of the deformed sheet was $0.41 \mathrm{~mm}$ and the strains were all above the forming limit diagram. It means the fracture defect could be avoided. In addition, the height of the flange conformed to the target goal to be achieved. However, this process produced a critical defect of wrinkling, as shown in Fig. 4(d), on the flange of hinge, which induces a problem in the subsequent trimming operation. Hence, even though the two-operation stamping process solved the fracture problem at the corner of the bottom and the flange of

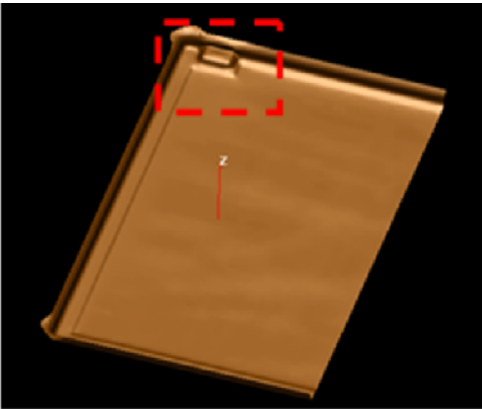

(b)

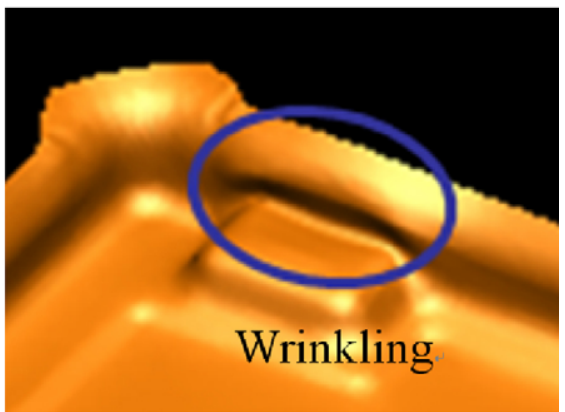

(d)

Fig. 4 - Two-operation stamping process. (a) Formation of sidewalls, (b) formation of hinges, (c) thickness distribution and (d) wrinkle. 


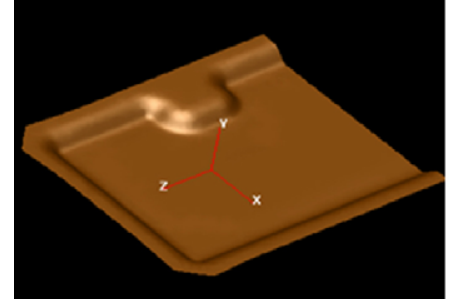

(a)

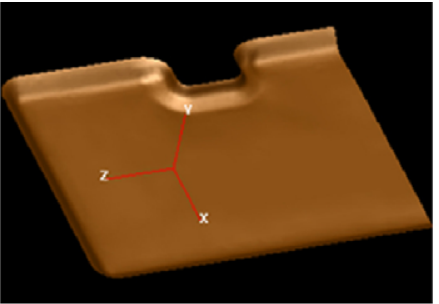

(b)

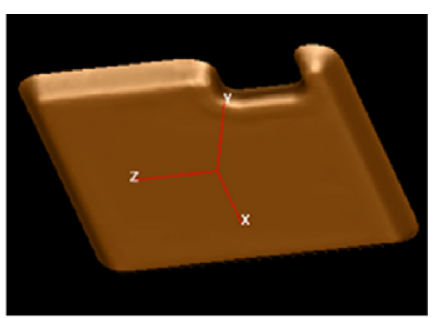

(c)

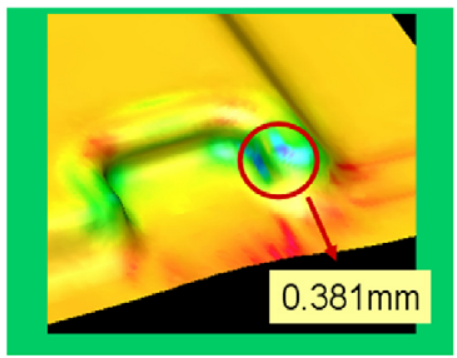

(d)

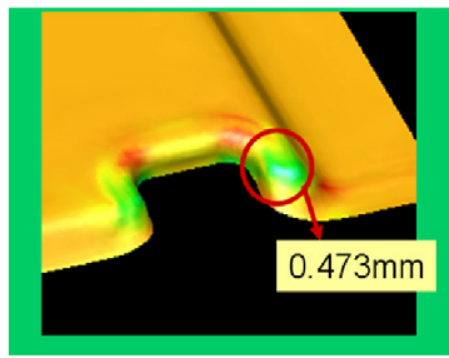

(e)

Fig. 5 - Four-operation stamping process. (a) First operation, (b) second operation, (c) third operation, (d) without trimming and (e) with trimming.

hinge, a better forming process is still expected to solve the wrinkling of flange of hinge.

\subsection{Four-operation stamping process}

The four-operation forming process proposed in the present study starts with the forming of three sidewalls and the flange of the hinge with a generous corner radius, as shown in Fig. 5(a). Since the sidewall close to the flange was open and the corner radius was larger than the desired ones, the flange was successfully formed without fracture. Such process success- fully avoided the difficulty of forming two geometric features simultaneously, but increased the material flow of the blank sheet. The next step was to trim the blank outside the sidewalls, and to calibrate the corner radius of $4 \mathrm{~mm}$ to the desired value of $2.5 \mathrm{~mm}$. The hinge was thus formed, as shown in Fig. 5(b). The third step was to fold the open side, so that the sidewall could be completed around its periphery, as shown in Fig. 5(c). The effect of trimming the extra sheet outside the sidewalls in the second step on the third step was studied. When the extra sheet was not trimmed, the thickness at the corner was $0.381 \mathrm{~mm}$, as shown in Fig. 5(d). The thickness of

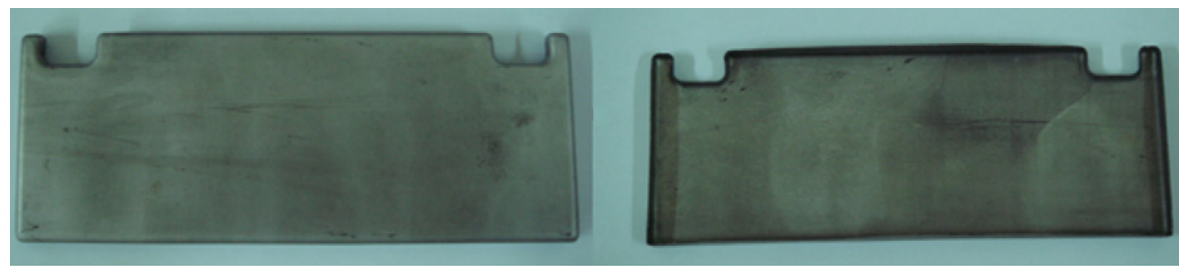

(a)

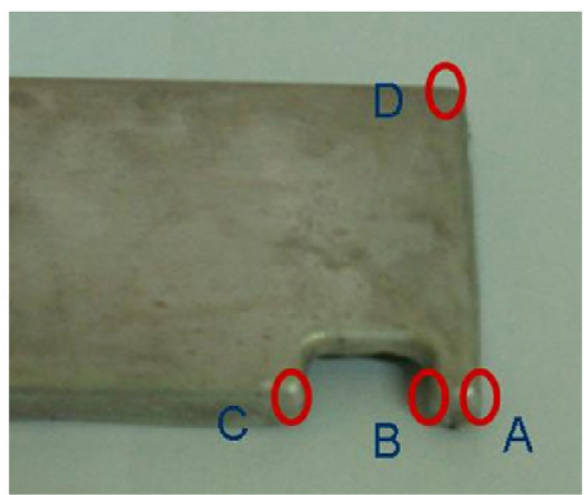

(b)

Fig. 6 - The sound product. (a) Without fracture and wrinkle and (b) locations of thickness measured. 
Table 1 - Comparison of thickness measured

\begin{tabular}{lllll} 
& \multicolumn{1}{c}{ A } & \multicolumn{1}{c}{ B } & \multicolumn{1}{c}{ C } & \multicolumn{1}{c}{ D } \\
\hline Experiment & $0.42 \mathrm{~mm}$ & $0.44 \mathrm{~mm}$ & $0.49 \mathrm{~mm}$ & $0.53 \mathrm{~mm}$ \\
Simulation & $0.423 \mathrm{~mm}$ & $0.448 \mathrm{~mm}$ & $0.508 \mathrm{~mm}$ & $0.532 \mathrm{~mm}$ \\
Error & $0.71 \%$ & $1.79 \%$ & $3.54 \%$ & $0.38 \%$ \\
\hline
\end{tabular}

the corner increased to $0.473 \mathrm{~mm}$, as shown in Fig. 5(e), if the trimming was implemented in the second step. The excessive material produced by the folding process in the third step was then trimmed off according to the parts design. The last step was the striking process that is applied to calibrate all the corner radii to the designed values. The minimum thickness at the corner of the final product was $0.42 \mathrm{~mm}$, and all the strains were above the forming limit diagram. It is to be noted that Fig. $5(\mathrm{a}-\mathrm{c})$ only shows the formation of one hinge. The same design concept was then extended to the stamping process of the complete top cover case.

\section{Experimental validation}

In order to validate the finite element analysis, an actual fouroperation stamping process was conducted with the use of $0.6 \mathrm{~mm}$ thick LZ91 sheet as the blank. The blank dimension and the tooling geometries were designed according to the finite element simulation results. A sound product without fracture and wrinkle was then manufactured, as shown in Fig. 6(a). To further validate the finite element analysis quantitatively, the thickness at the corners around the hinge of the sound product, as shown in Fig. 6(b), were measured and compared with those obtained from the finite element simulations, as listed in Table 1. It is seen in Table 1 that the experimental data and the finite element results were consistent. The four-operation process design based on the finite element analysis was then confirmed by the experimental data.

\section{Concluding remarks}

The press forming of magnesium alloy sheets was studied in the present study using the experimental approach and the finite element analysis. The formability of both AZ31 and LZ sheets was examined first. The research results indicated that the LZ91 sheet has favorable formability at room temperature, which is similar to that of AZ31 sheet at the forming temperature of $200^{\circ} \mathrm{C}$.

The superior formability of LZ91 sheet at room temperature was also demonstrated in the present study by successful manufacturing of the notebook top cover case. The proposed four-operation process lends itself to an efficient approach to form the hinge in the notebook with fewer operational procedures than that required in the current practice.

It also confirms that the notebook cover cases can be produced with LZ91 magnesium alloy LZ91 sheet by the stamping process. It provides an alternative to the electronics industry in the application of magnesium alloys.

\section{Acknowledgments}

The authors would like to thank the National Science Council of the Republic of China for financially supporting this research under the Project No. NSC-95-2622-E-002-019-CC3, which made this research possible. They would also like to thank ESI, France for the help in running the PAM_STAMP program.

\section{REFERENCES}

Chen, F.K., Huang, T.B., 2003. Formability of stamping magnesium-alloy AZ31 sheets. J. Mater. Process. Technol. 142, 643-647.

Chen, F.K., Huang, T.B., Chang, C.K., 2003. Deep drawing of square cups with magnesium alloy AZ31 sheets. Int. J. Mach. Tools Manuf. 43, 1553-1559.

Drozd, Z., Trojanová, Z., Kúdela, S., 2004. Deformation of behavior of Mg-Li-Al alloy. J. Mater. Compd. 378, 192-195.

Takuda, H., Yoshii, T., Hatta, N., 1999a. Finite-element analysis of the formability of a magnesium-based alloy AZ31 sheet. J. Mater. Process. Technol. 89/90, 135-140.

Takuda, H., Kikuchi, S., Tsukada, T., Kubota, K., Hatta, N., 1999b. Effect of strain rate on deformation behavior of a $\mathrm{Mg}-8.5 \mathrm{Li}-1 \mathrm{Zn}$ alloy sheet at room temperature. Mater. Sci. Eng. 271, 251-256. 